\title{
Dry Formulations Enhanced Mucoadhesive Properties and Reduced Cold Chain Handing of Influenza Vaccines
}

\author{
Nattika Saengkrit, ${ }^{1}$ Somsak Saesoo, ${ }^{1}$ Noppawan Woramongkolchai, ${ }^{1}$ Warayuth Sajomsang, ${ }^{1}$ Sarunya Phunpee, ${ }^{1}$ \\ Tararaj Dharakul, ${ }^{2}$ and Uracha Rungsardthong Ruktanonchai ${ }^{1,3}$
}

Received 25 April 2018; accepted 7 September 2018; published online 26 September 2018

\begin{abstract}
To alleviate concerns in health security, emergency flu vaccine stockpiles are required for ensuring rapid availability of vaccines when needed. Cold chain preservation, at high cost and risk, is necessary to maintain vaccine efficacy. This study aimed to develop a dry, easily storable formula for influenza vaccine preparation. The formulation with mucoadhesive properties is expected to facilitate rapid delivery via nasal administration. Chitosan, a cationic polymer, was used as cryo-protectant and to promote mucoadhesion. Optimal concentrations and molecular weights of chitosan polymers were screened, with short chain chitosan (10 kDa) being most suitable. H1N1 dry powder, in different formulations, was prepared via freeze-drying. A series of cryo-protectants, trehalose (T), chitosan (C), fetal bovine serum (FBS; F), or a combination of these (TCF), were screened for their effects on prolonging vaccine shelf life. Physicochemical monitoring (particle size and zeta potential) of powders complexed with mucin revealed that the order of cryoprotectant mixing during preparation was of critical importance. Results indicated that the TCF formula retains its activity up to 1 year as indicated by TCID $_{50}$ analysis. This approach was also successful at prolonging the shelf life of $\mathrm{H} 3 \mathrm{~N} 2$ vaccine, and has the potential for large-scale implementation, especially in developed countries where long-term storage of vaccines is problematic.
\end{abstract}

KEY WORDS: dry formulation; vaccine formulation; influenza vaccine; mucoadhesive; nasal vaccine delivery.

\section{INTRODUCTION}

Annual vaccination is the single best way to protect against flu illnesses, and these afford a good (60-90\% efficacy) degree of protection (1). Two types of influenza vaccines are currently available: inactivated (killed) and attenuated. At present, the most widely used influenza vaccines are inactivated types, applied through an intramuscular (i.m.) injection. However, live attenuated influenza vaccine (LAIV) is now also available and is gaining in interest due to its cost, ease of administration and induction of mucosal, as well as systemic immunity (2-4). LAIV is administrated by the pulmonary route in the form of a nasal spray, with the advantage that both local and cell-mediated immune responses are invoked $(5,6)$. A commercially available formulation, FluMist ${ }$, a cold-adapted live attenuated

\footnotetext{
${ }^{1}$ National Nanotechnology Center (NANOTEC), National Science and Technology Development Agency (NSTDA), Pathumthani, 12120, Thailand.

${ }^{2}$ Department of Immunology, Faculty of Medicine, Siriraj Hospital, Mahidol University, Bangkok, 10700, Thailand.

${ }^{3}$ To whom correspondence should be addressed. (e-mail: uracha@nanotec.or.th)
}

influenza vaccine, has been used for several decades in Russia (7) and has been approved in the USA for vaccination of healthy individuals (aged 5-49) since 2003. This LAIV formulation contains a sucrose-phosphate-glutamate stabilizer with the strain being cold-adapted such that it must be stored frozen $\left(-15\right.$ to $\left.-25^{\circ} \mathrm{C}\right)$ prior to use and should not be refrozen after thawing, to maintain product stability (8). Therefore, the cold chain is very important for maintaining LAIV product quality. Unfortunately, large quantities of vaccines are discarded each year due to expiration or cold chain interruption, which complicates stockpiling and is economically wasteful. Therefore, developing methodologies for stabilizing vaccines to reduce the cold chain is a major endeavor (9-11).

The most general method for stabilizing biopharmaceutical agents, such as proteins, DNA/RNA, and vaccines, is to prepare them as dry powders. In the solid dry form, biopharmaceutical agents are in a reduced or immobile state. Potential degradation pathways, such as hydrolysis, are also less likely under these conditions (10). However, drying and freezing can generate stress in samples, resulting in loss of structural integrity. Minimizing such damage can be addressed by selecting appropriate stabilizers for formulations 
(11). Cryo-preservative agents are used to protect LAIV, and these compounds protect the coated protein against structural changes, fusion/aggregation, membrane damage caused by ice crystals, intracellular ice formation, and $\mathrm{pH}$ shifts $(12,13)$. The mechanism of how these protect live viruses is not fully understood, such that trial-and-error screening is utilized for selection of effective cryo-protectant agents (14). Several classes of compounds, including carbohydrates, sugar alcohols, and animal-derived components are used as vaccine stabilizers. Disaccharides (i.e., sucrose and trehalose) are routinely employed although sorbitol and animal-derived components are also common (15-17). Trehalose, one of the most popular cryo-protectants used for biomolecule preservation (17-19), has the ability to remain amorphous during freeze-drying. During the drying step, trehalose can hydrogen bond with the coating protein or the lipid cell membrane of viruses, resulting in cell stabilization (18). At low temperatures, trehalose can prevent membranes from undergoing phase transitions during rehydration. Nevertheless, the advantages of trehalose are not universal, with a combination of lactalbumin hydrolysate and sucrose being a superior thermal protectant for live, attenuated peste des petits ruminants (PPR) vaccine (19). Similarly, a mixture of trehalose-amino acid and divalent cations affords better protection to a Camelpox virus vaccine than a sucrose/lactalbumine hydrolysate combination (20). For animal-derived components, gelatin and albumin are often used although major concerns of using these are potential dangers arising from prion contamination (21). Accordingly, plant-derived components have become the preferred option (22). Aside from these mainstream cryo-protectants, safe and economically feasible alternatives are being explored. Chitosan, for example, is used in vaccine development as an adjuvant or a nanocarrier for delivery of actives, especially in DNA vaccines (23-26). It also possesses mucoadhesive properties, opening up many potential uses for this compound in the biomedical and pharmaceutical fields.

Most inactivated influenza vaccines are stable for approximately 1 year at $4^{\circ} \mathrm{C}$; however, LAIV strictly requires cold storage $\left(-15\right.$ to $\left.-20^{\circ} \mathrm{C}\right)(7)$. To overcome this problem, this study aimed to develop a refrigerator stable LAIV formulation through the application of dry powder technology. The developed formula exhibited stability under conditions of refrigeration, while having mucoadhesive properties enabling a better immune response. The dry powder developed herein was characterized in terms of particle morphology and physiology, and the mucoadhesive properties confirmed. Storage stability was monitored for a period of 1 year at $4^{\circ} \mathrm{C}$, being evaluated in terms of vaccine efficacy. Results indicate that this formulation approach may be logistically attractive, allowing for alleviation of cold chain issues allowing substantial annual cost savings.

\section{MATERIALS AND METHODS}

\section{Chemicals}

Fetal bovine serum (FBS) was obtained from Biochrom AG (Berlin, Germany). Trypsin-EDTA, L-glutamine, penicillin $\mathrm{G}$ sodium, streptomicin sulfate, and amphotericin B were obtained from Invitrogen Corp. (NY, USA). Mucin type
III, extracted from porcine stomach, was obtained from Sigma-Aldrich (MO, USA). Dulbecco's Modified Eagle's Medium (DMEM) and MTT [3-(4,5-dimethylthiazol-2-yl)2,5-diphenyltetrazolium bromide] were obtained from GIBCO (NY, USA). Trehalose was bought from Mana Chemical (Bangkok, Thailand). Branched polyethylenimine (PEI; molecular weight $25 \mathrm{kDa}$ ) was purchased from SigmaAldrich (MO, USA). Chitosan polymers having molecular weights $(\mathrm{Mw})$ of $10 \mathrm{kDa}$ and $22 \mathrm{kDa}$ were purchased from OilZac Technologies Co., Ltd. (Bangkok, Thailand) while $600 \mathrm{kDa}$ chitosan was purchased from Seafresh Chitosan (Lab) Co., Ltd. (Chumphon, Thailand). Degrees of deacetylation (DD) in chitosan polymers were determined to be between 94 and $98 \%$ by ${ }^{1} \mathrm{H}$-NMR spectroscopy (27). Anti-influenza A was obtained from Merck (Darmstadt, Germany) and goat anti-mouse IgG was from KPL (MD, USA).

\section{Influenza Virus Propagation in Embryonated Chicken Eggs}

Influenza A virus, $\mathrm{H} 1 \mathrm{~N} 1$ and $\mathrm{H} 3 \mathrm{~N} 2$ were isolated and identified from clinical specimens at Department of Immunology, Faculty of Medicine Siriraj Hospital, Mahidol University. Influenza virus was propagated in embryonated chicken eggs to obtain consistent yields of viral stock. This involved viral propagation in pathogen-free fertilized chicken eggs (7-12 days post-fertilization). After location of a nonveined area of the allantoic cavity just below the air sac in each egg, inoculation with virus was done through a small hole in the shell, using a tuberculin syringe. After injection of virus into the allantoic cavity, the hole was sealed with melted paraffin. Eggs were incubated at $37^{\circ} \mathrm{C}$ for 3-7 days to allow the replication of virus and release into the allantoic fluid, the shell and chorioallantoic membranes were punctured with a pipette. The removed allantoic viral suspension was stored at $-80^{\circ} \mathrm{C}$ for further use.

\section{Screening and Optimization of Chitosan Polymers for Viral Preservation}

The quantity of chitosan used in formulations needed to be thoroughly optimized to avoid toxic effects of short chain chitosans on MDCK cells, which are used to determine viral infection efficacy. MDCK cells were cultured in DMEM with $10 \%$ fetal calf serum, 100 units $/ \mathrm{ml}$ penicillin, and $100 \mathrm{~g} / \mathrm{ml}$ streptomycin at $37^{\circ} \mathrm{C}$ with $5 \% \mathrm{CO}_{2}$. Treatment of MDCK cells with various molecular weight $(10,22$, and $600 \mathrm{kDa})$ chitosans at a range of concentrations $(0.35,0.7,1 \% \mathrm{w} / \mathrm{v})$ allowed for optimization of cell viability. Cell viability assays were performed $24 \mathrm{~h}$ after incubation according to the assay kit manufacturer's recommendations, with percentage cell viabilities expressed in relation to untreated (control) cells. A branched cationic polymer, PEI, was also employed in formulations for comparison.

\section{Preparation of Viral Dry Powders}

Viral dry powders, prepared by freeze-drying mixtures of virus suspensions with different cryo-protective agents, were obtained as follows. The control formulations were prepared by individually adding of $10 \% \mathrm{w} / \mathrm{v}$ trehalose, $1 \% \mathrm{w} / \mathrm{v}$ 
chitosan, and 10\% FBS into virus suspension (TCID $4.0 \times 10^{4} /$ $\mathrm{ml})$. The samples were mixed well by pipetting.

In order to verify the significance of the order of mixing and mucoadhesive property, the samples were prepared with and without the order of mixing. $10 \% w / v$ trehalose, $1 \% w / v$ chitosan, and $10 \%$ FBS were randomly added into virus suspension and mixed well by pipetting. Regarding the order of mixing, virus solution was mixed with $10 \%$ trehalose and $10 \% \mathrm{FBS}$, then added into $1 \% \mathrm{w} / v$ chitosan solution.

All samples were then subjected to freeze-drying with pre-cooling to $-196^{\circ} \mathrm{C}$ by using liquid nitrogen. After freezedrying for $24 \mathrm{~h}$ at $-80^{\circ} \mathrm{C}$ (pressure $0.1 \mathrm{mbar}$ ), the dry powders were obtained and stored at either $-80^{\circ} \mathrm{C}$ or $4^{\circ} \mathrm{C}$, for further analysis.

\section{Physical Characterization of Dry Viral Powder}

Scanning electron microscopy (SEM) (S-3400 N, Hitachi, Japan) was used to elucidate dry powder morphologies. Samples were placed onto carbon tape, which was then glued to an alumina stub. After coating with Au using a sputter coater under vacuum, samples were subjected to SEM imaging.

\section{Mucoadhesive Testing}

The in vitro mucoadhesive properties of re-suspended dry powder were evaluated according to the protocol of Takeuchi et al. (28). For testing, colloidal mucin was prepared in particulate form. Specifically, $1 \% w / v$ mucin type III was added to Tris buffer ( $\mathrm{pH}$ 6.8) and the mixture stirred continuously overnight at $37^{\circ} \mathrm{C}$. The mucin particle size was then reduced to $200-300 \mathrm{~nm}$ by ultrasonication (VCX750, Sonics \& Materials, Inc., CT USA). Centrifugation at $4000 \mathrm{rpm}$ for $20 \mathrm{~min}$ allowed separation of colloidal mucin from larger aggregates. For mucoadhesion tests, $1 \%(w / v)$ mucin suspension was mixed with various quantities of viral powder suspension, such that each suspension contained $v / v$ ratios of powder: mucin as followed; 0:1, 1:1, 2:1, 4:1, 6:1, 8:1, 10:1, 15:1, 20:1, and 100:1. The particle size and zeta potential values of each suspension were then measured using dynamic light scattering (DLS) (NanoZS4700 nanoseries, Malvern Instruments, Malvern, UK).

\section{Investigation of Viral Infectious Dose of Dry Formulation after Preservation}

H1N1 virus was utilized during infectious dose investigations. The viral invasion was observed in MDCK cells, allowing evaluations of the vaccine potency as a result of active virus infection. Freeze-dried vaccine was tested at set time points: $0,0.5,1,2,3,6,8,10$, and 12 months. For evaluations, MDCK cell suspensions were plated in 96-well plates, each well containing $100 \mu \mathrm{l}$ of DMEM with $10 \%$ fetal bovine serum at a density of $1.5 \times 10^{5}$ cells $/ \mathrm{ml}$. When confluence was reached (typically $24-48 \mathrm{~h}$ after plating), the viral suspension was added, affording a serial dilution. The mixture was then incubated at $37^{\circ} \mathrm{C}$ overnight or until the cytopathic effect (CPE) was observed. ELISA was then performed to determine the infectious titer of influenza virus with storage time, at $50 \%$ endpoint (median tissue infectious dose; $\mathrm{TCID}_{50}$ ) values. Anti-influenza A and goat anti-mouse IgG were employed as first and second ELISA antibodies. $\mathrm{TCID}_{50}$ values and $\mathrm{TCID}_{50}$ assay end points were calculated using the protocol of Reed and Muench (29). Titers obtained from three independent tests were analyzed statistically using one-way ANOVA. The above protocol was also successfully demonstrated in H3N2, another viral subtype.

\section{RESULTS}

\section{Optimization of Chitosan for Formulation}

This study aimed to develop a dried formulation for vaccine possessing with mucoadhesive properties. Chitosan was selected as a candidate polymer for this approach, with screening undertaken to ascertain a suitable molecular weight for use in formulations. MDCK cells were treated with 10, 22, and $600 \mathrm{kDa}$ chitosan at various concentrations $(0.35,0.7$, and $1 \% w / v)$ to evaluate their toxicity profiles. Results in Fig. 1 indicate that all chitosans exhibit favorable toxicity profiles, with cell viabilities greater than $80 \%$. Moreover, $10 \mathrm{kDa}$ chitosan appears to induce cell growth, consistent with previous observations (30). Considering the effects of molecular weight on cell viability, it is likely that 22 and $600 \mathrm{kDa}$ chitosan are more toxic than short chain of $10 \mathrm{kDa}$ chitosan. As a result, $1 \% w / v 10 \mathrm{kDa}$ chitosan was selected for formulations, and its toxicity was compared with a commercially available PEI (polyethylenimine), a mucoadhesive cationic polymer used in medical studies (Fig. 2). Branched chain PEI (MW 25 and $60 \mathrm{kDa}$ ) were selected in this study. MDCK cells were exposed to both chitosan (10 kDa) and PEI at concentrations of $0.002 \%, 0.025 \%$, and $1 \% w / v$. MTT assays revealed that toxicity of PEI is dose-dependent, and $1 \% w / v$ PEI induces significant cell death. However, chitosan exhibited no effects on cell viability, justifying its incorporation into the dry vaccine formulation.

\section{Study of the Significance of Mixing Order and Mucoadhesive Property Test}

Trehalose, FBS, and chitosan were selected as cryoprotectant candidates. The order of mixing on mucoadhesive test was examined. The interaction between vaccine and mucin particles was investigated by using the sub-micron mucin assay. Control mucin particles, prepared with size and

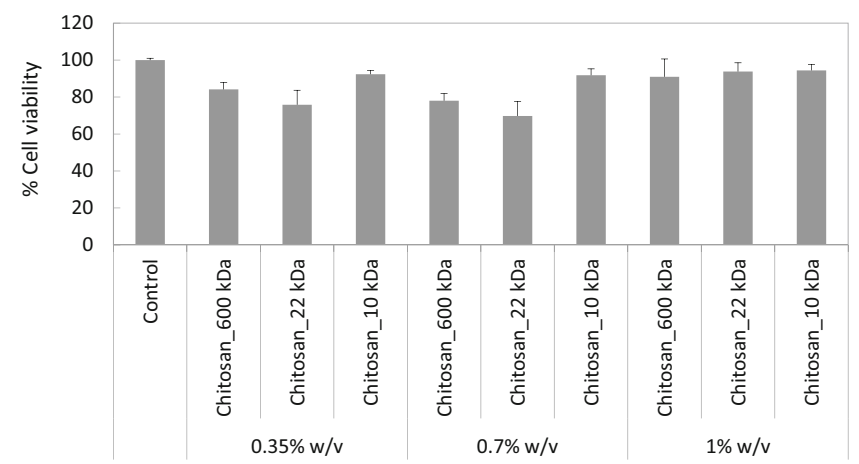

Fig. 1. Optimization of chitosan for formulation. Chitosan with molecular weight at 600,22 , and $10 \mathrm{kDa}$ were screened by measuring the cell viability assay with MDCK cells 


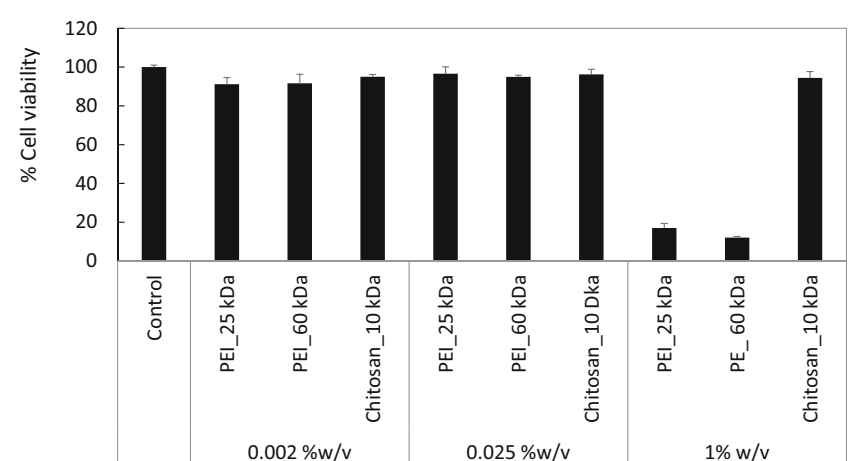

Fig. 2. The chitosan with $10 \mathrm{kDa}$ was tested on cell viability in comparison with a commercially available cationic polymer polyethylenimine (formed with branched 60 and $25 \mathrm{kDa}$ )

zeta potentials in the ranges of $260-280 \mathrm{~nm}$ and $-21.50 \pm$ $0.05 \mathrm{mV}$, respectively, were mixed with vaccine suspensions (at various concentrations), and surface properties (zeta potential and particle size) simultaneously monitored on DLS. Naked H1N1, $10 \mathrm{kDa}$ chitosan, and TCF vaccine were exposed to mucin. Particle sizes and zeta potentials for these complexes are highlighted in Fig. 3. Accordingly, the effect of the order of mixing on the vaccine formation was observed through particle size and zeta potential measurements, enabling physicochemical changes of the powder and mucin complexes at various ratios to be observed (Fig. 3a, b). Particle sizes and zeta potentials of formulated samples prepared with, and without, a set order of mixing, were compared with those using naked freeze-dried H1N1. The
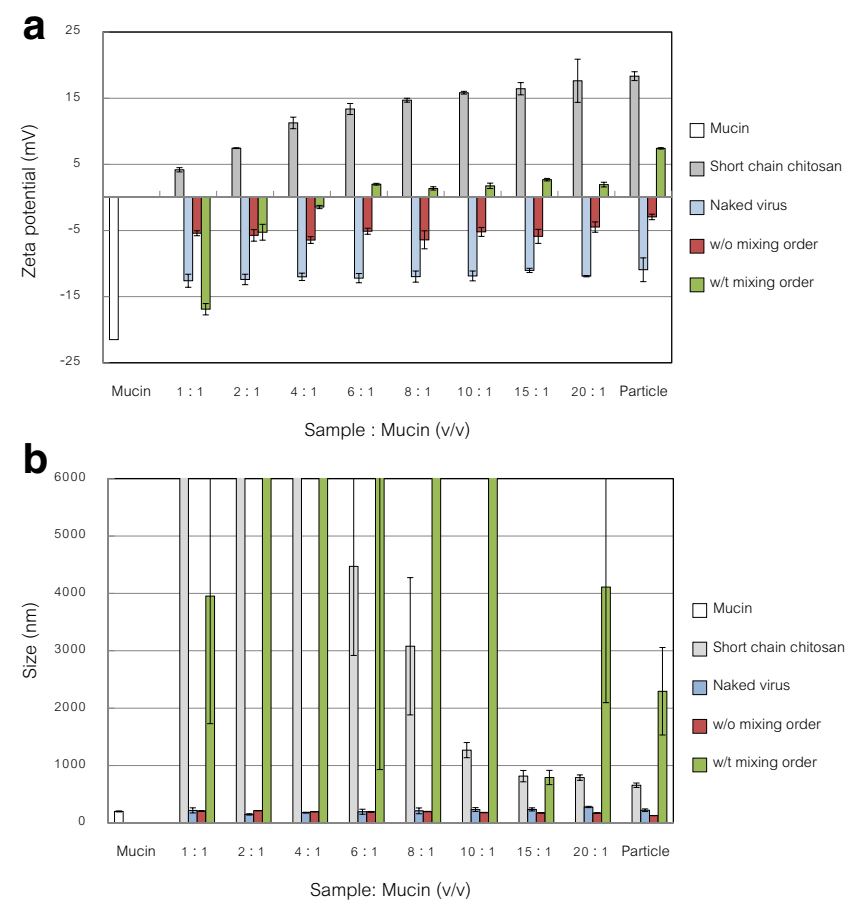

Fig. 3. The study of effect of order of mixing in vaccine preparation and mucoadhesive assay. Samples were prepared with and without a set of order mixing and compared with mucin, short chain chitosan, and naked freeze-dried virus. The charge of zeta potential values (a) and sizes (b) of sample and mucin complexes were monitored absence of size and zeta potential changes indicates that the binding of mucin with H1N1 could not be elevated. Formulations prepared using a set order of mixing exhibited changes in size and zeta potential, which were dependent on virus concentration. Binding enhancements of TCF-formulated H1N1 with mucin were thus clearly confirmed, with a virus:mucin binding have started binding via electrostatic interaction at the ratio of $0.05-0.15: 1 \mathrm{w} / \mathrm{w}$ and reached plateau of neutralization at $0.2-0.5: 1 \mathrm{w} / \mathrm{w}$. On the other hand, formulations prepared with random component mixing showed no binding with mucin due to no change of zeta potential value, underlining the criticality of mixing order in vaccine formulation preparation. The manufacturing of dry powder for flu LAIV vaccines should, therefore, be mixing sequence dependent, inducing layer-by-layer deposition on viral particles. Trehalose and FBS are first added to allow insertion of cryo-protectant into the viral membrane. Subsequent chitosan coating results in the desired mucoadhesive properties.

As for the mucoadhesive analysis, we found that naked H1N1 particles exhibit negative zeta potential $(-11.53 \pm$ $0.38 \mathrm{mV}$ ) arising from the lipid membrane, which does not significantly effect on mixing with mucin (Fig. 3a). Additionally, no change in particle size was found, suggesting that mucin-virus complexation does not occur (Fig. 3b). Short chain chitosan $(1 \%)$ formed complexes with mucin in a dosedependent manner, as indicated by a change of zeta potential and size. An addition of TCF to mucin resulted in dynamic changes in zeta potential, with the extent of surface positive charge being dependent on viral powder concentration. This result clearly implies affinity of the viral powder for mucin, illustrating the mucoadhesive nature of the TCF dry formulation.

\section{Morphology Observations Using SEM}

The morphology of vaccine powders derived from freeze-dried H1N1, freeze-dried H1N1 mixed with trehalose, FBS or chitosan, and combination of these (TCF) were revealed using SEM. Freeze-dried H1N1 exhibits an amorphous structure (Fig. 4), although mixing with trehalose results in spheroidal particles as a result of virus:trehalose complex formation. Addition of FBS, or short chain chitosan alone results in clump-like particles of virus and cryoprotectant complexes in which it is likely that the virus is coated with FBS or chitosan. However, TCF addition results in spherical clusters of H1N1 coated with protective layers: required attributes for influenza viral vaccine powders.

\section{Evaluation of Vaccine Stability on Storage}

Reducing the necessity of cold storage and prolonging shelf life are important goals when deriving vaccine formulations. Through the TCF formulation, in addition to obtaining mucoadhesive properties, we also aimed to increase the vaccine shelf life, allowing storage at $4^{\circ} \mathrm{C}$. To investigate this, influenza A virus $\mathrm{H} 1 \mathrm{~N} 1$ formulations were subjected to freeze-drying followed by $\mathrm{TCID}_{50}$ monitoring over time, as this relates to vaccine efficacy on preservation. Viral formulations were stored at $4^{\circ} \mathrm{C}$ for a year to investigate their shelf life. Naked viral suspensions were stocked at $-80^{\circ} \mathrm{C}$ and $4^{\circ} \mathrm{C}$, 


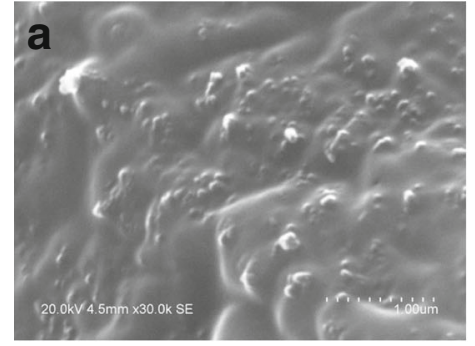

H1N1 freeze dried powder

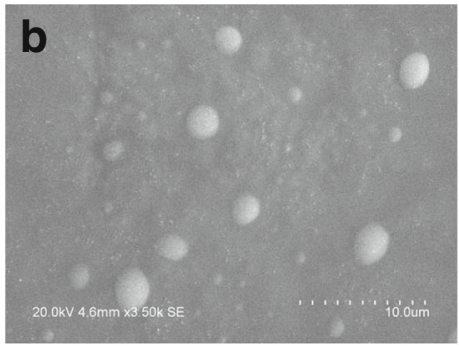

H1N1 freeze dried with trehalose

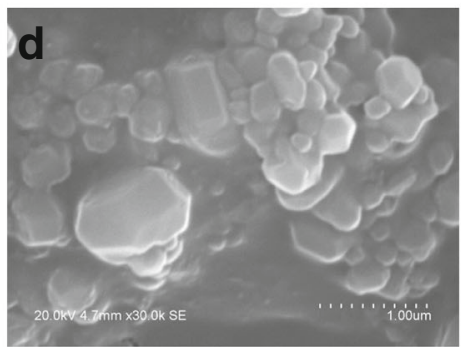

H1N1 freeze dried with short chain chitosan

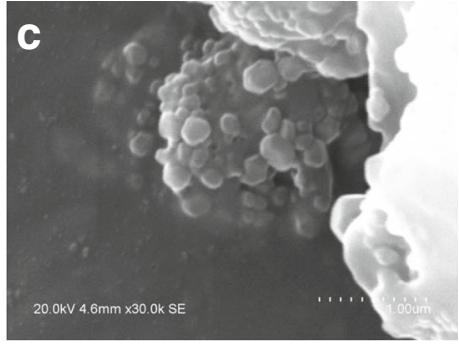

H1N1 freeze dried with FBS

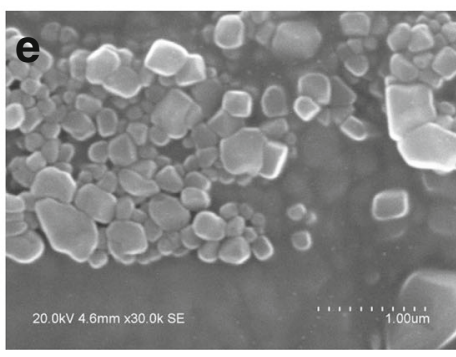

$\mathrm{H} 1 \mathrm{~N} 1$ freeze dried with

trehalose, FBS and short chain chitosan

Fig. 4. SEM micrographs of freeze-dried H1N1 (a), freeze-dried H1N1 mixed with $10 \% w / v$ of trehalose (b), freeze-dried H1N1 mixed with $10 \% w / v$ FBS (c), freeze-dried H1N1 mixed with $1 \% w / v$ of short chain chitosan (d), and freeze-dried H1N1 mixed with protectants; $10 \% w / v$ of trehalose, $10 \% w / v$ FBS, and $1 \% w / v$ of short chain chitosan (e)

as controls. The efficacy of freeze-dried virus, preserved using trehalose, FBS, chitosan, or TCF, were compared through periodic $\mathrm{TCID}_{50}$ monitoring after $0,0.5,1,2,6,8$, and 12 months. As indicated in Fig. 5, the efficacy of control virus was maintained on storage at $-80^{\circ} \mathrm{C}$; however, storage at $4^{\circ} \mathrm{C}$ results in a time dependent loss in activity. Virus preserved in trehalose showed slight reductions in infectious dose on storage for 12 months at $4^{\circ} \mathrm{C}$ indicative of some protection; however, FBS and chitosan formulations were not effective cryo-protectants under refrigeration conditions. However, TCF formulations exhibited excellent protective effects, with $\mathrm{TCID}_{50}$ values being comparable, even after 12 months, to those of frozen $\left(-80^{\circ} \mathrm{C}\right)$ naked virus. These observations and the mucoadhesive properties of the TCF formulation may allow for its effective stockpiling, and render it appropriate for nasal delivery against pandemic influenza viruses.

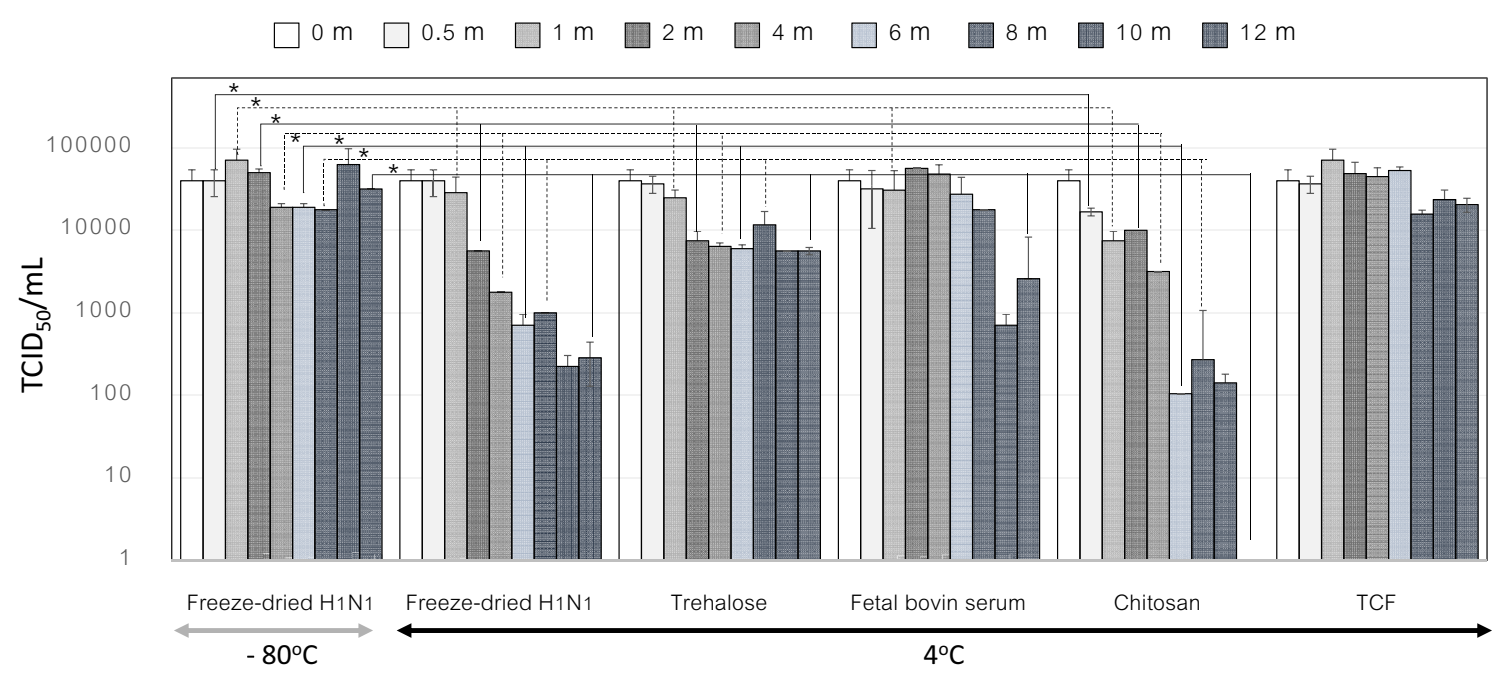

Fig. 5. Stability of the H1N1 during preservation up to 12 months at $-80^{\circ} \mathrm{C}$ and $4^{\circ} \mathrm{C}$. Viral survivals were tested by evaluation of TCID50. Samples are as followed; freeze-dried H1N1 (naked virus) mixed with $10 \% w / v$ of trehalose (Trehalose), freeze-dried H1N1 mixed with $10 \% w / v$ FBS (Fetal bovine serum), freeze-dried H1N1 mixed with $1 \% w / v$ of short chain chitosan (Chitosan), and freeze-dried H1N1 mixed with all protectants; $10 \% w / v$ of trehalose, $10 \% w / v$ FBS, and $1 \% w / v$ of short chain chitosan (TCF). The significant statistical difference $(*)$ was calculated using ANOVA (one-way analysis of variance) 


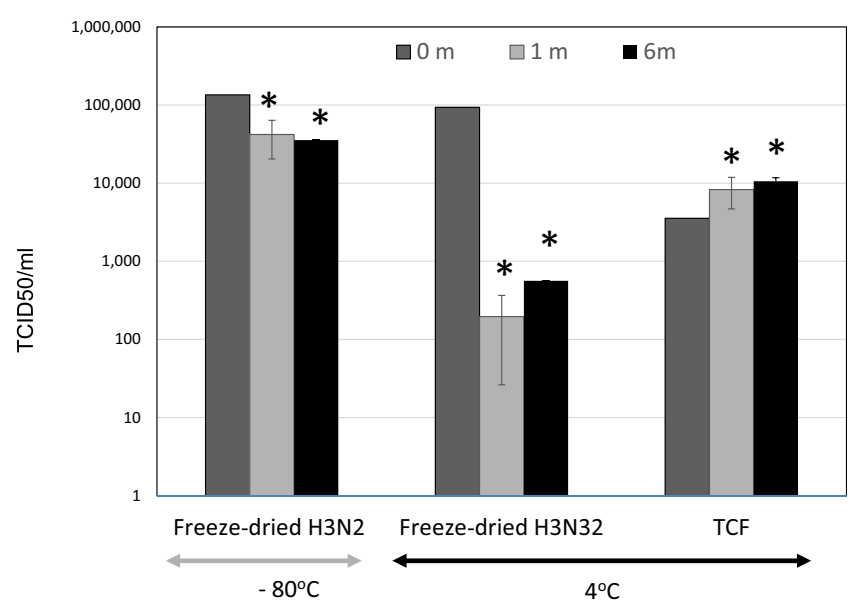

Fig. 6. Stability of the H3N2 during preservation up to 6 months at $80^{\circ} \mathrm{C}$ and $4^{\circ} \mathrm{C}$. Viral survivals were tested by evaluation of TCID50. Samples are preserved in the mix of $10 \% \mathrm{w} / \mathrm{v}$ of trehalose, $10 \% \mathrm{w} / \mathrm{v}$ FBS, and $1 \% w / v$ of short chain chitosan (TCF). The significant statistical difference $(*)$ was calculated using ANOVA

\section{Protocol Implementation to Other Influenza Viruses}

To verify whether the TCF protocol can be implemented for preservation of other influenza viruses, we selected $\mathrm{H} 3 \mathrm{~N} 2$ as a model system. In the same manner as used for $\mathrm{H} 1 \mathrm{~N} 1$, TCF formulated virus was preserved at $4^{\circ} \mathrm{C}$ and compared with naked freeze-dried $\mathrm{H} 3 \mathrm{~N} 2$ preserved at -80 and $4^{\circ} \mathrm{C}$. Viral survival was determined though the determination of infectious dose, with $\mathrm{TCID}_{50}$ being evaluated periodically over 6 months (Fig. 6). As in H1N1, naked virus loses efficacy rapidly when stored at $4^{\circ} \mathrm{C}$, but is maintained on deep freezing $\left(-80^{\circ} \mathrm{C}\right)$. TCF proved remarkably effective for H3N2 cryo-protection: dramatic improvements in virus preservation efficiency are evident on comparison with freezedried naked virus. This result is encouraging, indicating that TCF could be a major step forward in freezing reduction of cold chain for preservation of LAIV vaccines, and other viral preparations.

\section{CONCLUSIONS}

This work describes the development of a protective agent for LAIV vaccines which overcomes the freezing requirement in cold chain preservation. The H1N1 virus formulation possesses mucoadhesive properties for potential nasal delivery. The formulation is composed of three stabilizers, and can be applied to produce freeze-dried H1N1 in a dry powder form. Trehalose was chosen as viral membrane stabilizer and cryo-protectant during freezing, FBS is a nutrient promoting viral re-activation, while short chain chitosan is believed to work synergistically with trehalose as a coating agent. The order of mixing during the formulation process proved critical, especially in terms of mucoadhesion. Survival monitoring of the $\mathrm{H} 1 \mathrm{~N} 1$ preservation at $4^{\circ} \mathrm{C}$ over 12 months found no loss of activity, with viral stability comparable to naked virus preserved at $-80^{\circ} \mathrm{C}$. This research is a step forward in vaccine preservation protocols, being cheap and effective, and could be potentially implemented to address concerns of vaccine security and health care, especially in developing countries.

\section{FUNDING INFORMATION}

This research was financially supported by National Nanotechnology Center (NANOTEC), Thailand.

\section{REFERENCES}

1. Cox R, Brokstad K, Ogra P. Influenza virus: immunity and vaccination strategies. Comparison of the immune response to inactivated and live, attenuated influenza vaccines. Scand J Immunol. 2004;59(1):1-15.

2. Amorij J-P, Hinrichs WLJ, Frijlink HW, Wilschut JC, Huckriede A. Needle-free influenza vaccination. Lancet Infect Dis. 2010;10(10):699-711.

3. Lu D, Hickey AJ. Pulmonary vaccine delivery. Expert Rev Vaccines. 2007:6(2):213-26.

4. Sullivan VJ, Mikszta JA, Laurent P, Huang J, Ford B. Noninvasive delivery technologies: respiratory delivery of vaccines. Expert Opin Drug Deliv. 2006;3(1):87-95.

5. Block SL, Yogev R, Hayden FG, Ambrose CS, Zeng W, Walker RE. Shedding and immunogenicity of live attenuated influenza vaccine virus in subjects 5-49 years of age. Vaccine. 2008;26(38):4940-6.

6. Greenbaum E, Engelhard D, Levy R, Schlezinger M, Morag A, Zakay-Rones Z. Mucosal (SIgA) and serum (IgG) immunologic responses in young adults following intranasal administration of one or two doses of inactivated, trivalent anti-influenza vaccine. Vaccine. 2004;22(20):2566-77.

7. Milstien JB et al. Temperature sensitivity of vaccines. 2006.

8. Yannarell DA, Goldberg KM, Hjorth RN. Stabilizing coldadapted influenza virus vaccine under various storage conditions. J Virol Methods. 2002;102(1):15-25.

9. Murugappan S, Patil HP, Kanojia G, ter Veer W, Meijerhof T, Frijlink HW, et al. Physical and immunogenic stability of spray freeze-dried influenza vaccine powder for pulmonary delivery: comparison of inulin, dextran, or a mixture of dextran and trehalose as protectants. Eur J Pharm Biopharm. 2013;85(3):716-25.

10. Amorij J, et al. Development of stable influenza vaccine powder formulations: challenges and possibilities. Pharm Res. 2008;25(6):1256-73.

11. Block SL, Reisinger KS, Hultquist M, Walker RE, for the CAIV-T Study Group. Comparative immunogenicities of frozen and refrigerated formulations of live attenuated influenza vaccine in healthy subjects. Antimicrob Agents Chemother. 2007;51(11):4001-8.

12. Bhatnagar BS, Bogner RH, Pikal MJ. Protein stability during freezing: separation of stresses and mechanisms of protein stabilization. Pharm Dev Technol. 2007;12(5):505-23.

13. Salo R, Cliver D. Effect of acid pH, salts, and temperature on the infectivity and physical integrity of enteroviruses. Arch Virol. 1976;52(4):269-82.

14. Hansen L, et al. Freeze-drying of live virus vaccines: a review. Vaccine. 2015;33(42):5507-19.

15. Tannock GA, Hierholzer JC, Bryce DA, Chee CF, Paul JA. Freeze-drying of respiratory syncytial viruses for transportation and storage. J Clin Microbiol. 1987;25(9):1769-71.

16. Sarkar J, Sreenivasa BP, Singh RP, Dhar P, Bandyopadhyay SK. Comparative efficacy of various chemical stabilizers on the thermostability of a live-attenuated peste des petits ruminants (PPR) vaccine. Vaccine. 2003;21(32):4728-35.

17. Zhai S, Hansen RK, Taylor R, Skepper JN, Sanches R, Slater NKH. Effect of freezing rates and excipients on the infectivity of a live viral vaccine during lyophilization. Biotechnol Prog. 2004;20(4):1113-20.

18. Crowe LM, Crowe JH, Rudolph A, Womersley C, Appel L. Preservation of freeze-dried liposomes by trehalose. Arch Biochem Biophys. 1985;242(1):240-7. 
19. Kumru OS, Joshi SB, Smith DE, Middaugh CR, Prusik T, Volkin DB. Vaccine instability in the cold chain: mechanisms, analysis and formulation strategies. Biologicals. 2014;42(5):237-59.

20. Prabhu M, Bhanuprakash V, Venkatesan G, Yogisharadhya R, Bora DP, Balamurugan V. Evaluation of stability of live attenuated camelpox vaccine stabilized with different stabilizers and reconstituted with various diluents. Biologicals. 2014;42(3):169-75.

21. Nemoto T, Horiuchi M, Ishiguro N, Shinagawa M. Detection methods of possible prion contaminants in collagen and gelatin. Arch Virol. 1999;144(1):177-84.

22. Chun B-H, Lee YK, Lee BC, Chung N. Development of a varicella virus vaccine stabilizer containing no animal-derived component. Biotechnol Lett. 2004;26(10):807-12.

23. Prego C, Paolicelli P, Díaz B, Vicente S, Sánchez A, GonzálezFernández Á, et al. Chitosan-based nanoparticles for improving immunization against hepatitis B infection. Vaccine. 2010;28(14):2607-14.

24. Sawaengsak C, Mori Y, Yamanishi K, Srimanote P, Chaicumpa $\mathrm{W}$, Mitrevej A, et al. Intranasal chitosan-DNA vaccines that protect across influenza virus subtypes. Int $\mathrm{J}$ Pharm. 2014;473(1):113-25.
25. Zhao K, Shi X, Zhao Y, Wei H, Sun Q, Huang $\mathrm{T}$, et al. Preparation and immunological effectiveness of a swine influenza DNA vaccine encapsulated in chitosan nanoparticles. Vaccine. 2011;29(47):8549-56.

26. Heffernan MJ, Zaharoff DA, Fallon JK, Schlom J, Greiner JW. In vivo efficacy of a chitosan/IL-12 adjuvant system for proteinbased vaccines. Biomaterials. 2011;32(3):926-32.

27. Lavertu M, Xia Z, Serreqi AN, Berrada M, Rodrigues A, Wang $\mathrm{D}$, et al. A validated $1 \mathrm{H}$ NMR method for the determination of the degree of deacetylation of chitosan. J Pharm Biomed Anal. 2003;32(6):1149-58.

28. Takeuchi H, et al. Novel mucoadhesion tests for polymers and polymer-coated particles to design optimal mucoadhesive drug delivery systems. Adv Drug Deliv Rev. 2005;57(11):1583-94.

29. Reed LJ, Muench H. A simple method of estimating fifty per cent endpoints. Am J Epidemiol. 1938;27(3):493-7.

30. Rakkhithawatthana V, Sanitrum P, Sajomsang W, Na Ubon P, Tencomnao T, Saengkrit N. Investigation of gene transferring efficacy through nano-polyplex consisting of methylated N-(4pyridinylmethyl) chitosan chloride and poly (ethylenimine) in human cell lines. Carbohydr Polym. 2010;80(1):276-84. 